\title{
Soft theorems and the KLT-relation
}

\section{Rafael Aoude ${ }^{a}$ and Andreas Helset ${ }^{b}$}

${ }^{a}$ PRISMA ${ }^{+}$Cluster of Excellence $\&$ Institute of Physics, Johannes Gutenberg-Universität Mainz, 55099 Mainz, Germany

${ }^{b}$ Niels Bohr International Academy and Discovery Center, Niels Bohr Institute, University of Copenhagen, Blegdamsvej 17, DK-2100 Copenhagen, Denmark

E-mail: aoude@uni-mainz.de, ahelset@nbi.ku.dk

ABSTRACT: We find new relations for the non-universal part of the Yang-Mills amplitudes by combining the KLT-relation and the soft behavior of gauge and gravity amplitudes. We also extend the relations to include contributions from effective operators.

KEywords: Effective Field Theories, Scattering Amplitudes

ARXiv EPrint: 1911.09962 


\section{Contents}

1 Introduction 1

$\begin{array}{lll}2 & \text { BFCW } & 2\end{array}$

3 Soft theorem 4

4 KLT-relation $\quad 5$

5 Non-universal relations $\quad 6$

$\begin{array}{llr}6 & \text { Effective operators } & 7\end{array}$

$\begin{array}{lll}7 & \text { Conclusion } & 10\end{array}$

$\begin{array}{ll}\text { A Sub-subleading terms } & 11\end{array}$

B Sub-subleading terms from effective operator $\quad 12$

\section{Introduction}

The study of scattering amplitudes when the momentum of one or more particles becomes soft has a long history [1-12]. Weinberg showed that the scattering amplitudes factorize when a photon or graviton becomes soft, and that this factorization is universal $[9,10]$. The universality of the soft photon theorem is due to charge conservation, while for a soft graviton it follows from the equivalence principle. A subleading soft theorem for photons at tree-level was proven by Low [5]. Similar subleading soft theorems for gravitons and gluons have more recently been discussed using eikonal methods [13-15]. Also, Cachazo and Strominger showed that the sub-subleading soft graviton correction at tree-level is also universal [16].

The soft graviton theorem was shown to be connected to the Bondi, van der Burg, Metzner and Sachs (BMS) symmetry [17, 18], as a Ward identity [19-21]. This has sparked an interest in the connection between asymptotic symmetries and soft theorems (see ref. [22] for a list of references). The subleading soft theorem is known to be related to the supertranslations and superrotations for asymptotic symmetries and the sub-subleading soft theorem related symmetries was recently analysed in refs. [23, 24]. The authors found a new class of vector fields, which hints in the direction of a BMS algebra extension.

At loop level, the leading soft theorems for photons and gravitons remain unchanged. However, the loop corrections to the subleading soft theorems for gluons and gravitons were discussed in refs. [25, 26]. 
The soft behavior of scattering amplitudes when more than one particle is taken soft has also been studied (see e.g. ref. [27] and references therein). The soft theorems have also been discussed outside four dimensions [28-31] using the scattering equation framework by Cachazo, He, and Yuan (CHY) [32-34].

Much of the recent progress in calculating gravitational scattering amplitudes relies the connection between gauge amplitudes and gravity amplitudes [35, 36]. One manifestation of this connection is the Kawai-Lewellen-Tye (KLT) relations [37], which relates open and closed string amplitudes at tree level. In the field theory limit, the KLT-relation states that a sum of products of two color-ordered Yang-Mills amplitudes is a gravity amplitude.

In this paper, we study the connection between gravity and gauge soft theorems via the KLT-relations. We compare both sides of the formula at sub-subleading order in the soft-momentum expansion and obtain relations for the non-universal part of the YangMills amplitude. To the best of our knowledge, no other relations have been obtained for the non-universal piece of the Yang-Mills amplitudes. We further study the insertion of effective operators, which start contributing at sub-leading order. We also obtain relations for the non-universal effective amplitude at sub- and sub-subleading order.

The paper is organized as follows: Section 2 reviews the derivation of the soft theorems using Britto-Cachazo-Feng-Witten (BCFW) recursion relations. Section 3 presents the soft theorems of Yang-Mills and gravity amplitudes, while section 4 introduces the KLTrelations. Two of the new results of the paper are presented in section 5, where the soft limit of the amplitudes and the KLT-relation are used to find non-trivial relations which the Yang-Mills amplitudes must satisfy. An extension of these results is presented in section 6, when effective operators are included. We conclude in section 7 .

\section{BFCW}

We will review the derivation of the soft theorems for Yang-Mills and gravity amplitudes using the BCFW recursion relations $[38,39]$. This follows closely the derivation of the new soft theorems by Cachazo and Strominger [16]. We use the spinor-helicity formalism, with the convension $s_{a b}=\langle a, b\rangle[b, a]$. For an $(n+1)$-point amplitude with a soft particle $s$ with positive helicity, ${ }^{1} \mathcal{A}_{n+1}(s, 1, \ldots, n)$, we perform the BCFW shift

$$
\lambda_{s}(z)=\lambda_{s}+z \lambda_{n}, \quad \tilde{\lambda}_{n}(z)=\tilde{\lambda}_{n}-z \tilde{\lambda}_{s} .
$$

The original amplitude can be recovered from the complex deformed amplitude as the residue at $z=0$,

$$
\mathcal{A}_{n+1}=\frac{1}{2 \pi i} \int \frac{d z}{z} \mathcal{A}_{n+1}(z)
$$

Using Cauchy's residue theorem, we find the following relation

$$
\mathcal{A}_{n+1}=\sum_{\text {diagrams } I} \mathcal{A}_{L}\left(z_{I}\right) \frac{1}{P_{I}^{2}} \mathcal{A}_{R}\left(z_{I}\right)
$$

\footnotetext{
${ }^{1}$ The negative helicity case can be derived analogously.
} 
where the amplitude factorizes into a sum of two lower-point amplitudes, assuming the contribution from $|z| \rightarrow \infty$ vanishes. The product of the two amplitudes is the residue in Cauchy's theorem, therefore they are evaluated at the pole $z_{I}$. The pole is found for each diagram by solving the equation $P_{I}^{2}\left(z_{I}\right)=0$. From this relation we can build up higher-point amplitudes recursively.

The sum in equation (2.3) can be split into two parts as

$$
\mathcal{A}_{n+1}=\mathcal{A}_{3}\left(s\left(z_{I}\right), 1, I\right) \frac{1}{P_{I}^{2}} \mathcal{A}_{n}\left(-I, 2, \ldots, n\left(z_{I}\right)\right)+\mathcal{R}_{n+1} .
$$

The remainder term is written as

$$
\mathcal{R}_{n+1}=\sum_{\text {diagrams I }} \mathcal{A}_{L}\left(s\left(z_{I}\right), 1,2, \ldots, j\right) \frac{1}{P_{I}^{2}} \mathcal{A}_{R}\left(j+1, \ldots, n\left(z_{I}\right)\right)
$$

where $\mathcal{A}_{L}$ in equation (2.5) is a four- or higher-point amplitude. Under the holomorphic scaling $\lambda_{s} \rightarrow \epsilon \lambda_{s}, \tilde{\lambda}_{s} \rightarrow \tilde{\lambda}_{s}$, the amplitude ${ }^{2}$ for a soft gluon takes the form

$$
\begin{aligned}
\mathcal{A}_{n+1} & =\frac{1}{\epsilon^{2}} \frac{\langle n 1\rangle}{\langle n s\rangle\langle s 1\rangle} \mathcal{A}_{n}\left(\left\{\lambda_{1}, \tilde{\lambda}_{1}+\epsilon \frac{\langle n s\rangle}{\langle n 1\rangle} \tilde{\lambda}_{s}\right\}, \ldots,\left\{\lambda_{n}, \tilde{\lambda}_{s}+\epsilon \frac{\langle s 1\rangle}{\langle n 1\rangle} \tilde{\lambda}_{s}\right\}\right)+\mathcal{R}_{n+1} \\
& =\frac{1}{\epsilon^{2}} \frac{\langle n 1\rangle}{\langle n s\rangle\langle s 1\rangle} e^{\epsilon\left(\frac{\langle n s\rangle}{\langle n 1\rangle} \tilde{\lambda}_{s} \frac{d}{d \tilde{\lambda}_{1}}+\frac{\langle s 1\rangle}{\langle n 1\rangle} \tilde{\lambda}_{s} \frac{d}{d \tilde{\lambda}_{n}}\right)} \mathcal{A}_{n}+\mathcal{R}_{n+1} .
\end{aligned}
$$

This form was written down in ref. [40]. Also, the authors of ref. [16] showed that $\mathcal{R}_{n+1}$ is of order $\mathcal{O}\left(\epsilon^{0}\right)$. A similar expression can be written down for gravity, with the leading pole being $\mathcal{O}\left(\epsilon^{-3}\right)$. By expanding the exponential we can find the universal leading and (at tree-level) subleading soft factors, $S_{\mathrm{YM}}^{(0)}$ and $S_{\mathrm{YM}}^{(1)}$. The exponential contains derivative terms, for which we use the notation $\tilde{\nabla}_{a, b}=\tilde{\lambda}_{a}^{\dot{\alpha}} \frac{d}{d \tilde{\lambda}_{b}^{\dot{\alpha}}}$. The soft factors can be written as

$$
S_{\mathrm{YM}}^{(k)}=\frac{\langle n 1\rangle}{\langle n s\rangle\langle s 1\rangle} \frac{1}{k !}\left(\frac{\langle n s\rangle}{\langle n 1\rangle} \tilde{\nabla}_{s, 1}+\frac{\langle s 1\rangle}{\langle n 1\rangle} \tilde{\nabla}_{s, n}\right)^{k},
$$

where the soft factors with $k \geq 2$ give only a part of the amplitude. Again, a similar partial infinite soft factor for gravity can be found analogously.

A partial infinite soft theorem for effective operators can also be found using the same method. For Yang-Mills, we find that

$$
\bar{S}_{\mathrm{YM}}^{(k+1)}=-\left(\frac{[n s]}{\langle n s\rangle}+\frac{[1 s]}{\langle 1 s\rangle}\right) \frac{1}{k !}\left(\frac{\langle n s\rangle}{\langle n 1\rangle} \tilde{\nabla}_{s, 1}+\frac{\langle s 1\rangle}{\langle n 1\rangle} \tilde{\nabla}_{s, n}\right)^{k} .
$$

The $k=0$ term reproduces the leading soft theorem for effective operators with a spin-1 particle discussed in ref. [41]. The first contribution from effective operators for a YangMills amplitude appears at subleading order. For gravity, the first contribution from effective operators only enters at sub-subleading order.

\footnotetext{
${ }^{2}$ We will denote a Yang-Mills amplitude by $\mathcal{A}$, and a gravity amplitude by $\mathcal{M}$.
} 


\section{Soft theorem}

We will show the connection between the soft factors of gauge theory and gravity using the KLT-formula. In particular, at sub-subleading order in the soft expansion we find new relations between tree-level amplitudes. We start with an amplitude with one external soft particle. The soft limits of an amplitude with an external soft spin-1/spin-2 particle are

$$
\begin{aligned}
\mathcal{A}_{n+1} & =\left(\frac{S_{\mathrm{YM}}^{(0)}}{\epsilon^{2}}+\frac{S_{\mathrm{YM}}^{(1)}}{\epsilon}+S_{\mathrm{YM}}^{(2)}\right) \mathcal{A}_{n}+\mathcal{R}_{n+1}+\mathcal{O}\left(\epsilon^{1}\right), \\
\mathcal{M}_{n+1} & =\left(\frac{S_{\mathrm{GR}}^{(0)}}{\epsilon^{3}}+\frac{S_{\mathrm{GR}}^{(1)}}{\epsilon^{2}}+\frac{S_{\mathrm{GR}}^{(2)}}{\epsilon}\right) \mathcal{M}_{n}+\mathcal{O}\left(\epsilon^{0}\right),
\end{aligned}
$$

respectively. The $S_{\mathrm{YM}}^{(i)}$ is the $i$ th subleading soft factor of an amplitude with a soft spin-1 particle, and similarly for gravity. The non-universal part of the Yang-Mills amplitude enters at sub-subleading order. In the KLT-formula, two Yang-Mills amplitudes with different color-ordering are required: $\mathcal{A}_{n}(t, \sigma, n-1, n)$ and $\tilde{\mathcal{A}}_{n}(n-1, \rho, n, t)$. From now on we assume that whenever $\mathcal{A}_{n}$ and $\tilde{\mathcal{A}}_{n}$ is written, we have this particular ordering. The same holds for the $(n+1)$-point amplitudes; the relevant color-orderings are $\mathcal{A}_{n+1}(t, \sigma, n-1, n, n+1)$, $\tilde{\mathcal{A}}_{n+1}(n-1, \rho, n, t, n+1), \mathcal{R}_{n+1}(t, \sigma, n-1, n, n+1)$ and $\tilde{\mathcal{R}}_{n+1}(n-1, \rho, n, t, n+1)$. We leave the ordering implicit from now on.

The soft limit of the Yang-Mills amplitude is given by the soft factors in equation (2.7). The soft limit of the gravity amplitude is

$$
\begin{aligned}
\mathcal{M}_{n+1}(1,2, \ldots, n+1) & =-\frac{1}{\epsilon^{3}} \sum_{k=1}^{n} \frac{[n+1, k]}{\langle n+1, k\rangle} \\
& {\left[\frac{\langle x, k\rangle\langle y, k\rangle}{\langle x, n+1\rangle\langle y, n+1\rangle} \frac{\epsilon}{2}\left(\frac{\langle x, k\rangle}{\langle x, n+1\rangle}+\frac{\langle y, k\rangle}{\langle y, n+1\rangle}\right) \tilde{\nabla}_{n+1, k}+\frac{\epsilon^{2}}{2}\left(\tilde{\nabla}_{n+1, k}\right)^{2}\right] \mathcal{M}_{n}(1,2, \ldots, n), }
\end{aligned}
$$

where $x, y$ are reference spinors which specifies a gauge. The amplitudes are independent of the choice of $x, y$. Some care is needed when implementing the momentum conservation. We use $(n+1)$-point and $n$-point momentum conservation, given by

$$
\tilde{\lambda}_{i}=-\sum_{\substack{k=1 \\ k \neq i, j}}^{n} \frac{\langle j, k\rangle}{\langle j, i\rangle} \tilde{\lambda}_{k}-\epsilon \frac{\langle j, n+1\rangle}{\langle j, i\rangle} \tilde{\lambda}_{n+1}, \quad \tilde{\lambda}_{j}=-\sum_{\substack{k=1 \\ k \neq i, j}}^{n} \frac{\langle i, k\rangle}{\langle i, j\rangle} \tilde{\lambda}_{k}-\epsilon \frac{\langle i, n+1\rangle}{\langle i, j\rangle} \tilde{\lambda}_{n+1} .
$$

and

$$
\tilde{\lambda}_{i}=-\sum_{\substack{k=1 \\ k \neq i, j}}^{n} \frac{\langle j, k\rangle}{\langle j, i\rangle} \tilde{\lambda}_{k}, \quad \tilde{\lambda}_{j}=-\sum_{\substack{k=1 \\ k \neq i, j}}^{n} \frac{\langle i, k\rangle}{\langle i, j\rangle} \tilde{\lambda}_{k}
$$

We use eq. (3.4) for all $(n+1)$-point amplitudes $\mathcal{A}_{n+1}$ and $\mathcal{M}_{n+1}$, and eq. (3.5) for all $n$-point amplitudes $\mathcal{A}_{n}$ and $\mathcal{M}_{n}$, with $i, j=n-1, n$. Also, the total derivatives in the soft factors are

$$
\tilde{\nabla}_{s, k}=\tilde{\lambda}_{s}^{\dot{\alpha}} \frac{d}{d \tilde{\lambda}_{k}^{\dot{\alpha}}}=\tilde{\lambda}_{s}^{\dot{\alpha}}\left[\frac{\partial}{\partial \tilde{\lambda}_{k}^{\dot{\alpha}}}+\left(-\frac{\langle j, k\rangle}{\langle j, i\rangle}\right) \frac{\partial}{\partial \tilde{\lambda}_{i}^{\dot{\alpha}}}+\left(-\frac{\langle i, k\rangle}{\langle i, j\rangle}\right) \frac{\partial}{\partial \tilde{\lambda}_{j}^{\dot{\alpha}}}\right] .
$$


When first using momentum conservation before applying the soft factors, the total derivatives reduce to partial derivatives.

\section{KLT-relation}

In string theory, the KLT-relation provides a connection between open and closed string amplitudes. In the limit of infinite string tension, field theory is recoved and a relation between gravity and gauge amplitudes is obtained. Once all the proper permutations are taken into account, the KLT-relation gives the gravity amplitude as the "square" of the gauge amplitudes. For low-point amplitudes, the formulas are relatively simple, which helps streamlining gravitational scattering-amplitude calculations.

The most general form of the KLT-relation is [42, 43]

$$
\begin{gathered}
\mathcal{M}_{n}(1,2, \ldots, n)=(-1)^{n+1} \sum_{\sigma \in S_{n-3}} \sum_{\alpha \in S_{j-1}} \sum_{\beta \in S_{n-2-j}} \mathcal{A}_{n}\left(1, \sigma_{2, j}, \sigma_{j+1, n-2}, n-1, n\right) \\
\times \mathcal{S}\left[\alpha_{\sigma(2), \sigma(j)} \mid \sigma_{2, j}\right]_{p_{1}} \mathcal{S}\left[\sigma_{j+1, n-2} \mid \beta_{\sigma(j+1), \sigma(n-2)}\right]_{p_{n-1}} \tilde{\mathcal{A}}_{n}\left(\alpha_{\sigma(2), \sigma(j)}, 1, n-1, \beta_{\sigma(j+1), \sigma(n-2)}, n\right),
\end{gathered}
$$

where $\alpha, \beta, \sigma, \rho$ are particular orderings of the color-ordered Yang-Mills amplitudes. The KLT-kernel $\mathcal{S}$ is defined as

$$
\mathcal{S}\left[i_{1}, \ldots, i_{k} \mid j_{1}, \ldots, j_{k}\right]_{p_{1}}=\prod_{t=1}^{k}\left(s_{i_{t} 1}+\sum_{q>t}^{k} \theta\left(i_{t}, i_{q}\right) s_{i_{t} i_{q}}\right),
$$

where $\theta\left(i_{a}, i_{b}\right)$ is 0 if $i_{a}$ sequentially comes before $i_{b}$ in the set $\left\{j_{1}, \ldots, j_{k}\right\}$, and otherwise it takes the value 1 . One of the properties of the kernel is to take into account the fact that Yang-Mills amplitudes are color-order while gravity amplitudes are not. It was also proven in ref. [43] that the KLT-relation, as written in eq. (4.1), is independent of the choice of $j$. Therefore, with $j=2$, we have for an $(n+1)$-point amplitude that

$$
\begin{gathered}
\mathcal{M}_{n+1}(1,2, \ldots, n, n+1)=(-1)^{n} \sum_{t=1}^{n-2} \sum_{\sigma, \rho \in S_{n-3}} \mathcal{A}_{n+1}(t, \sigma, n-1, n, n+1) \mathcal{S}[t \mid t]_{p_{n+1}} \mathcal{S}[\sigma \mid \rho]_{p_{n-1}} \\
\times \tilde{\mathcal{A}}_{n+1}(n-1, \rho, n, t, n+1) .
\end{gathered}
$$

In the next section, we are going to apply the soft theorems for each amplitude and collect terms at different orders in $1 / \epsilon$. Thus, we also need the soft limit of the KLT-kernel, which is $[40]$

$$
\mathcal{S}[t \mid t]_{p_{n+1}} \mathcal{S}[\sigma \mid \rho]_{p_{n-1}} \rightarrow \epsilon s_{t, n+1} e^{\epsilon \frac{\langle n, n+1\rangle}{\langle n, t\rangle} \tilde{\nabla}_{n+1, t}} \mathcal{S}[\sigma \mid \rho]_{p_{n-1}}
$$

We also have the $S_{n-3}$-symmetric form of the KLT-relation for $n$-point amplitudes

$$
\mathcal{M}_{n}(1,2, \ldots, n)=(-1)^{n+1} \sum_{\sigma, \rho \in S_{n-3}} \mathcal{A}_{n}(1, \sigma, n-1, n) \mathcal{S}[\sigma \mid \rho]_{p_{n-1}} \tilde{\mathcal{A}}_{n}(1, n-1, \rho, n) .
$$

The different forms of the KLT-relation will be useful shortly. 


\section{Non-universal relations}

The usual procedure when using the KLT-relation is to obtain gravity amplitudes from Yang-Mills amplitudes, since usually the Yang-Mills amplitudes are easier to calculate. Here, we go in the opposite direction. We use information about the gravity amplitudes to obtain relations on the Yang-Mills side. As we noted before, the non-universal part of the Yang-Mills amplitude enters at sub-subleading order in the soft-momentum expansion. At this order, we also have a universal part which comes from an exponential of the associated soft factor. Both terms contribute in the KLT-formula. On the other hand, gravity contains only universal pieces at $\mathcal{O}(1 / \epsilon)$. We equate the soft limit of the gravity amplitude with the soft limit of the Yang-Mills side in the KLT-relation. This immediately gives constraints for the non-universal part of the Yang-Mills amplitudes. We describe the procedure in the following and give a detailed derivation in appendix A.

We use the KLT-relation in equation (4.3), which we write as

$$
\mathcal{M}_{n+1}=\sum_{\sigma, \rho} \mathcal{A}_{n+1}(\sigma) \mathcal{S}_{n+1}[\sigma \mid \rho] \tilde{\mathcal{A}}_{n+1}(\rho)
$$

For the left-hand side of equation (5.1), we apply the soft-graviton theorem in equation (3.3), and then apply the KLT-relation in equation (4.5) for each $k$ in the sum for the soft factor,

$$
\begin{aligned}
\mathcal{M}_{n+1} & =\left(\frac{S_{\mathrm{GR}}^{(0)}}{\epsilon^{3}}+\frac{S_{\mathrm{GR}}^{(1)}}{\epsilon^{2}}+\frac{S_{\mathrm{GR}}^{(2)}}{\epsilon}\right) \mathcal{M}_{n} \\
& =\left(\frac{S_{\mathrm{GR}}^{(0)}}{\epsilon^{3}}+\frac{S_{\mathrm{GR}}^{(1)}}{\epsilon^{2}}+\frac{S_{\mathrm{GR}}^{(2)}}{\epsilon}\right) \sum_{\alpha, \beta} \mathcal{A}_{n}(\alpha) \mathcal{S}_{n}[\alpha \mid \beta] \tilde{\mathcal{A}}_{n}(\beta) .
\end{aligned}
$$

The right-hand side of equation (5.1) becomes

$$
\begin{aligned}
\sum_{\sigma, \rho} \mathcal{A}_{n+1} \mathcal{S}_{n+1}[\sigma \mid \rho] \tilde{\mathcal{A}}_{n+1}=\sum_{\sigma, \rho} & {\left[\left(\frac{S_{\mathrm{YM}}^{(0)}}{\epsilon^{2}}+\frac{S_{\mathrm{YM}}^{(1)}}{\epsilon}+S_{\mathrm{YM}}^{(2)}\right) \mathcal{A}_{n}+\mathcal{R}_{n+1}\right] \mathcal{S}_{n+1}[\sigma, \rho] } \\
\times & {\left[\left(\frac{S_{\mathrm{YM}}^{(0)}}{\epsilon^{2}}+\frac{S_{\mathrm{YM}}^{(1)}}{\epsilon}+S_{\mathrm{YM}}^{(2)}\right) \tilde{\mathcal{A}}_{n}+\tilde{\mathcal{R}}_{n+1}\right] }
\end{aligned}
$$

when we use the soft limit of the Yang-Mills amplitudes in equation (2.7). We can match the left-hand side and the right-hand side of equation (5.1) at each order in 1/ $\epsilon$. A detailed analysis of the relation at order $1 / \epsilon^{3}$ and $1 / \epsilon^{2}$ was performed in ref. [40], resulting in new relations for the KLT-kernel.

Focusing on $\mathcal{O}(1 / \epsilon)$, we find simple relations between the universal and non-universal piece of the Yang-Mills amplitudes. A more detailed derivation can be found in appendix A. 
The non-universal pieces are defined as

$$
\begin{aligned}
& R_{1}=(-1)^{n+1} \sum_{t=1}^{n-2} \sum_{\sigma, \rho \in S_{n-3}} \frac{1}{\epsilon} \frac{[t, n+1]\langle t, n-1\rangle}{\langle n+1, n-1\rangle} \mathcal{R}_{n+1}^{\epsilon \rightarrow 0} \mathcal{S}[\sigma \mid \rho]_{p_{n-1}} \tilde{\mathcal{A}}_{n}, \\
& R_{2}=(-1)^{n+1} \sum_{t=1}^{n-2} \sum_{\sigma, \rho \in S_{n-3}} \frac{1}{\epsilon} \frac{[t, n+1]\langle n, t\rangle}{\langle n+1, n\rangle} \mathcal{A}_{n} \mathcal{S}[\sigma \mid \rho]_{p_{n-1}} \tilde{\mathcal{R}}_{n+1}^{\epsilon \rightarrow 0},
\end{aligned}
$$

while the universal pieces come from second derivatives,

$$
\begin{aligned}
& T_{1}=(-1)^{n} \sum_{t=1}^{n-2} \sum_{\sigma, \rho \in S_{n-3}} \frac{1}{\epsilon} \frac{[t, n+1]\langle n, n-1\rangle}{\langle t, n\rangle\langle n+1, n-1\rangle}\left[\frac{1}{2} \tilde{\nabla}_{n+1, t}^{2}\left(\mathcal{A}_{n} \mathcal{S}[\sigma \mid \rho]_{p_{n-1}}\right)\right] \tilde{\mathcal{A}}_{n}, \\
& T_{2}=(-1)^{n} \sum_{t=1}^{n-2} \sum_{\sigma, \rho \in S_{n-3}} \frac{1}{\epsilon} \frac{[t, n+1]\langle n-1, n\rangle}{\langle t, n-1\rangle\langle n+1, n\rangle} \mathcal{A}_{n} \mathcal{S}[\sigma \mid \rho]_{p_{n-1}}\left[\frac{1}{2} \tilde{\nabla}_{n+1, t}^{2} \tilde{\mathcal{A}}_{n}\right] .
\end{aligned}
$$

The relation between the universal and non-universal pieces of the Yang-Mills amplitudes is

$$
T_{1}+T_{2}=R_{1}+R_{2}
$$

Equation (5.8) is a new, non-trival relation, illustrating that the non-universal part of the Yang-Mills amplitude possesses some hidden structure. Remarkably, find that the relation simplifies into two parts, ${ }^{3}$ given by

$$
\begin{aligned}
& T_{1}=R_{1}, \\
& T_{2}=R_{2} .
\end{aligned}
$$

Explicitly, for e.g. equation (5.10), this means that

$$
\sum_{t=1}^{n-2} \sum_{\sigma, \rho \in S_{n-3}} \frac{1}{\epsilon} \mathcal{A}_{n} \mathcal{S}[\sigma \mid \rho]_{p_{n-1}}\left[\frac{[t, n+1]\langle n, n-1\rangle}{\langle t, n\rangle\langle n+1, n-1\rangle}\left[\frac{1}{2} \tilde{\nabla}_{n+1, t}^{2} \tilde{\mathcal{A}}_{n}\right]+\frac{[t, n+1]\langle n, t\rangle}{\langle n+1, n\rangle} \tilde{\mathcal{R}}_{n+1}^{\epsilon \rightarrow 0}\right]=0 .
$$

This is a non-trivial relation for $\mathcal{R}_{n+1}$, which previously have not been discussed. It relates the second derivative of the universal part of a $n$-point amplitude to the non-universal piece of the $(n+1)$-point amplitude. Taking as an example the $n+1=6 \mathrm{NMHV}$ amplitude. There are usually three BCFW-diagrams contributing to the amplitude. One of them contains all the universal soft behavior, as in equation (2.4), while the other two diagrams belongs to $\mathcal{R}_{n+1}$ (see equation (2.5)). In the soft momenta limit, $\epsilon \rightarrow 0$, we have a relation between these two diagrams and the second derivative of the 5-point amplitude $\mathcal{A}_{n}$.

\section{$6 \quad$ Effective operators}

The inclusion of effective operators in soft theorems were first studied in ref. [44]. The authors considered the operators $F^{3}, R^{3}$, and $R^{2} \phi$, and found that the soft theorems hold

\footnotetext{
${ }^{3}$ We have explicitly verified this through seven-points.
} 
for the two first operators while the soft graviton theorem receives a contribution from the last operator at sub-sub-leading order. More general operators were considered in ref. [41], where was shown that the soft theorem for a Yang-Mills particle is corrected at the subleading and sub-subleading order, while gravity amplitudes get corrections at the sub-subleading order. The modified soft theorems take the form

$$
\begin{aligned}
\mathcal{A}_{n+1} & =\left(\frac{S_{\mathrm{YM}}^{(0)}}{\epsilon^{2}}+\frac{S_{\mathrm{YM}}^{(1)}}{\epsilon}+S_{\mathrm{YM}}^{(2)}\right) \mathcal{A}_{n}+\left(\frac{\bar{S}_{\mathrm{YM}}^{(1)}}{\epsilon}+\bar{S}_{\mathrm{YM}}^{(2)}\right) \overline{\mathcal{A}}_{n}+\mathcal{R}_{n+1}+\mathcal{O}(\epsilon), \\
\mathcal{M}_{n+1} & =\left(\frac{S_{\mathrm{GR}}^{(0)}}{\epsilon^{3}}+\frac{S_{\mathrm{GR}}^{(1)}}{\epsilon^{2}}+\frac{S_{\mathrm{GR}}^{(2)}}{\epsilon}\right) \mathcal{M}_{n}+\frac{\bar{S}_{\mathrm{GR}}^{(2)}}{\epsilon} \overline{\mathcal{M}}_{n}+\mathcal{O}(\epsilon) .
\end{aligned}
$$

All amplitudes, including the remainder terms $\mathcal{R}_{n+1}$, can contain contributions from effective operators. The bar and superscript $(k)$ denote that, when corrected by effective operators, the particle $k$ of the $(n+1)$ - and $n$-point amplitudes may be of different particle type. The soft theorems for the effective operator corrections are

$$
\bar{S}_{\mathrm{YM}}^{(1)} \overline{\mathcal{A}}_{n}=-\sum_{k} \frac{[s, k]}{\langle s, k\rangle} \overline{\mathcal{A}}_{n}^{(k)}, \quad \bar{S}_{\mathrm{GR}}^{(2)} \overline{\mathcal{M}}_{n}=-\sum_{k} \frac{[s, k]^{3}}{\langle s, k\rangle} \overline{\mathcal{M}}_{n}^{(k)},
$$

where $s$ is the soft particle and $k$ is adjacent to the soft particle. We have absorbed the couplings into the amplitudes. The sum in equation (6.3) for a Yang-Mills particle goes over the two adjacent legs, while for gravity it sums over all other particles. The sub-subleading soft term for Yang-Mills particles can be found in eq. (2.8).

The second ingredient we need is the KLT-relation. The open-closed string KLTrelations are similar to the field-theory KLT-relations, with a different kernel. For instance, the 4-point string KLT-relation turns into

$$
\mathcal{M}_{4}^{\text {closed }}(1,2,3,4)=\mathcal{A}_{4}^{\text {open }}(1,2,3,4)\left[\frac{\kappa^{2}}{4 \pi \alpha^{\prime}} \sin (\pi x)\right] \tilde{\mathcal{A}}_{4}^{\text {open }}(1,2,4,3)
$$

where $x=-\alpha^{\prime} s_{12}$ and $\alpha^{\prime}$ is the inverse string tension. A generalized prescription for the KLT-relations for effective amplitudes was analysed in refs. [45-47]. The new, generalized kernel used in refs. [45-47] was organized as a Taylor expansion in powers of $\alpha^{\prime} s_{12}$,

$$
\frac{\sin (\pi x)}{\pi} \rightarrow x\left(1+c_{1} x+c_{2} x^{2}+\ldots\right)
$$

The first order in $\alpha^{\prime}$ recovers the usual KLT-kernel.

To consider the KLT-relation for effective amplitudes, we need to make some assumptions. First, we assume that a general $n$-point KLT-relation for effective amplitudes follows the structure found in refs. [45-47], i.e. the kernel is generalized, where the leading order reproduces the original kernel, and the kernel can be expanded as a Taylor expansion in powers of $s_{i j} / \Lambda^{2}$, where $\Lambda$ is some energy scale. In string theory, $\alpha^{\prime}$ takes the role of $1 / \Lambda^{2}$, as can be seen from equation (6.4). Second, we assume that the soft limit of the kernel is similar to eq. (4.4), with possibly more powers of $s_{t, n+1}$. Therefore, we assume that the 
soft limit of the kernel is

$$
\mathcal{S}[t \mid t]_{p_{n+1}} \mathcal{S}[\sigma \mid \rho]_{p_{n-1}} \rightarrow \epsilon s_{t, n+1}\left(\sum_{\ell=0}^{\infty} c_{\ell}\left(\epsilon s_{t, n+1}\right)^{\ell}\right) e^{\epsilon \frac{\langle n, n+1\rangle}{\langle n, t\rangle} \tilde{\nabla}_{n+1, t}} \mathcal{S}[\sigma \mid \rho]_{p_{n-1}},
$$

where $c_{0}=1$. We absorb any mass scale into the unknown coefficiencts $c_{\ell}$, such that the mass dimension of $c_{\ell}$ is $-2 \ell$.

The left-hand side of the KLT-relation in equation (5.1) now becomes

$$
\begin{aligned}
\mathcal{M}_{n+1} & =\left(\frac{S_{\mathrm{GR}}^{(0)}}{\epsilon^{3}}+\frac{S_{\mathrm{GR}}^{(1)}}{\epsilon^{2}}+\frac{S_{\mathrm{GR}}^{(2)}}{\epsilon}\right) \mathcal{M}_{n}+\left(\frac{\bar{S}_{\mathrm{GR}}^{(2)}}{\epsilon}\right) \overline{\mathcal{M}}_{n} \\
& =\left(\frac{S_{\mathrm{GR}}^{(0)}}{\epsilon^{3}}+\frac{S_{\mathrm{GR}}^{(1)}}{\epsilon^{2}}+\frac{S_{\mathrm{GR}}^{(2)}}{\epsilon}\right) \sum_{\alpha, \beta} \mathcal{A}_{n}(\alpha) \mathcal{S}_{n}[\alpha \mid \beta] \tilde{\mathcal{A}}_{n}(\beta)+\left(\frac{\bar{S}_{\mathrm{GR}}^{(2)}}{\epsilon}\right) \overline{\mathcal{M}}_{n} .
\end{aligned}
$$

Note that the only difference between equation (5.2) and equation (6.7) is the additional term coming from the effective-operator extension of soft-graviton theorem. The right-hand side of equation (5.1) is

$$
\begin{aligned}
& \sum_{\sigma, \rho}\left[\left(\frac{S_{\mathrm{YM}}^{(0)}}{\epsilon^{2}}+\frac{S_{\mathrm{YM}}^{(1)}}{\epsilon}+S_{\mathrm{YM}}^{(2)}\right) \mathcal{A}_{n}+\left(\frac{\bar{S}_{\mathrm{YM}}^{(1)}}{\epsilon}+\bar{S}_{\mathrm{YM}}^{(2)}\right) \overline{\mathcal{A}}_{n}+\mathcal{R}_{n+1}\right] \mathcal{S}_{n+1}[\sigma, \rho] \\
& \quad \times\left[\left(\frac{S_{\mathrm{YM}}^{(0)}}{\epsilon^{2}}+\frac{S_{\mathrm{YM}}^{(1)}}{\epsilon}+S_{\mathrm{YM}}^{(2)}\right) \tilde{\mathcal{A}}_{n}+\left(\frac{\bar{S}_{\mathrm{YM}}^{(1)}}{\epsilon}+\bar{S}_{\mathrm{YM}}^{(2)}\right) \tilde{\mathcal{\mathcal { A }}}_{n}+\tilde{\mathcal{R}}_{n+1}\right] .
\end{aligned}
$$

By equating equations (6.7) and (6.8) and comparing order-by-order in $1 / \epsilon$, we see that the first correction from the effective operators appears at subleading order for the right-hand side and at sub-subleading order for the left-hand side. Therefore, at order $\mathcal{O}\left(1 / \epsilon^{2}\right)$, we find the relation

$$
0=\bar{U}_{1}+\bar{U}_{2}+U_{3}+U_{4}
$$

where the first two terms are given by the modifications of the soft theorem for effective operators,

$$
\begin{aligned}
& \bar{U}_{1}=\frac{(-1)^{n+1}}{\epsilon^{2}} \sum_{t=1}^{n-2} \sum_{\sigma, \rho \in S_{n-3}} \frac{[t, n+1]\langle n, t\rangle}{\langle n, n+1\rangle} \mathcal{A}_{n} \mathcal{S}[\sigma \mid \rho]_{p_{n-1}}\left[\frac{[n+1, n-1]}{\langle n+1, n-1\rangle} \tilde{\mathcal{\mathcal { A }}}_{n}^{(n-1)}+\frac{[n+1, t]}{\langle n+1, t\rangle} \tilde{\overline{\mathcal{A}}}_{n}^{(t)}\right] \\
& \bar{U}_{2}=\frac{(-1)^{n+1}}{\epsilon^{2}} \sum_{t=1}^{n-2} \sum_{\sigma, \rho \in S_{n-3}}\left[\frac{[n+1, n]}{\langle n+1, n\rangle} \overline{\mathcal{A}}_{n}^{(n)}+\frac{[n+1, t]}{\langle n+1, t\rangle} \overline{\mathcal{A}}_{n}^{(t)}\right] \mathcal{S}[\sigma \mid \rho]_{p_{n-1}} \frac{[t, n+1]\langle t, n-1\rangle}{\langle n-1, n+1\rangle} \tilde{\mathcal{A}}_{n} .
\end{aligned}
$$

The third term comes from the expansion of the kernel, which has an unknown parameter $c_{1}$,

$$
U_{3}=\frac{(-1)^{n}}{\epsilon^{2}} \sum_{t=1}^{n-2} \sum_{\sigma, \rho \in S_{n-3}} c_{1} \frac{[t, n+1]^{2}\langle t, n\rangle\langle t, n-1\rangle}{\langle n, n+1\rangle\langle n+1, n-1\rangle} \mathcal{A}_{n} \mathcal{S}[\sigma \mid \rho]_{p_{n-1}} \tilde{\mathcal{A}}_{n} .
$$


The last term is

$$
U_{4}=\frac{(-1)^{n}}{\epsilon^{2}} \sum_{t=1}^{n-2} \sum_{\sigma, \rho \in S_{n-3}} \frac{[t, n+1]\langle n, n-1\rangle}{\langle n-1, n+1\rangle\langle n, n+1\rangle} \mathcal{A}_{n} \mathcal{S}[\sigma \mid \rho]_{p_{n-1}}\left[\tilde{\nabla}_{n+1, t} \tilde{\mathcal{A}}_{n}\right] .
$$

which was also found in the analysis in ref. [40], and was shown to vanish as long as the original kernel, defined in equation (4.2), satisfies two non-trivial identities. As we have not specified the generalized kernel fully, we keep this term for generality.

At $\mathcal{O}(1 / \epsilon)$, we proceed analogously. For the left-hand side of equation (5.1) we obtain the same terms $P_{1-6}$ in equations (A.1) to (A.6) in addition to the gravity effective operator term. For the right-hand side, we find the same $Q_{1-6}$ in equations (A.7) to (A.12) and $R_{1,2}$ in equations (5.4) and (5.5) as in section 5. The new contributions appear when we have used $\bar{S}_{\mathrm{YM}}^{(1)}, \bar{S}_{\mathrm{YM}}^{(2)}$, or higher terms in the expansion of the kernel. All the new contributions are given in appendix B.

After some manipulations, we obtain the following relation

$$
\frac{\bar{S}_{\mathrm{GR}}^{(2)}}{\epsilon} \overline{\mathcal{M}}_{n}+T_{1}+T_{2}=\bar{Q}+R_{1}+R_{2} .
$$

where $\bar{Q}$ is defined in the appendix. As expected, when the effective operators are turned off we recover equation (5.8). Although the relation equation (6.12) is more complicated than the relation for the original amplitudes (with no effective operators), we can still systematically organize the contributions from the effective operators into a simple formula. We found here the most general relations for the effective terms. However, further assumptions can be made on the type of effective operators that contribute to the amplitude and the form of the kernel, which can further simplify the relations. We leave this for a future investigation.

\section{Conclusion}

Using the KLT-relation and the soft limit of Yang-Mills and gravity amplitudes, we have found new, non-trivial relations for the sub-subleading part of the Yang-Mills amplitudes. Previous analysis has only considered the subleading terms, and the sub-subleading part has not previously been fully discussed. The new relations provide non-trivial constraints for the behavior of the Yang-Mills amplitudes under the soft limit. We also studied the analogous relations when contributions from effective operators are included.

The new relations give information about the non-universal part of the Yang-Mills amplitude. In obtaining the relations, we went in the oppositve direction of most of uses of the KLT-formula, where we made use of the behavior of the gravity amplitude to extract information for the Yang-Mills amplitude.

As we have used the spinor-helicity formalism, our results are restricted to four dimensions. Extending the analysis to arbitrary dimensions would provide insight into the generality of the result. A natural framework for studying the relations in arbitrary dimensions is the CHY-formalism. 
Recently, infinite partial soft theorems were discussed in refs. [48, 49]. Understanding the connection between our results and the infinite partial soft theorems would be illuminating. Also, non-linear relations for Yang-Mills amplitudes were presented in refs. [50, 51]. We leave the study of the connection between these non-linear relations and the relations presented in this paper as a future project.

\section{Acknowledgments}

We thank N. Emil J. Bjerrum-Bohr, Poul H. Damgaard, and Matt von Hippel for useful discussions. The work of A.H. was in part supported by the Danish National Research Foundation (DNRF91) and the Carlsberg Foundation. The work of R.A. was supported by the Alexander von Humboldt Foundation, in the framework of the Sofja Kovalevskaja Award 2016, endowed by the German Federal Ministry of Education and Research and also supported by the Cluster of Excellence "Precision Physics, Fundamental Interactions, and Structure of Matter" (PRISMA+ EXC 2118/1) funded by the German Research Foundation (DFG) within the German Excellence Strategy (Project ID 39083149).

\section{A Sub-subleading terms}

We outline the derivation of the relation for the non-universal terms at $\mathcal{O}(1 / \epsilon)$. Recall that the color-ordered amplitudes are given by $\mathcal{A}_{n} \equiv \mathcal{A}_{n}(t, \sigma, n-1, n)$ and $\tilde{\mathcal{A}}_{n} \equiv \tilde{\mathcal{A}}_{n+1}(n-$ $1, \rho, n, t)$ and the definition of the derivative operator is $\tilde{\nabla}_{a, b}=\tilde{\lambda}_{a}^{\dot{\alpha}} \frac{d}{d \tilde{\lambda}_{b}^{\dot{\alpha}}}$. The left-hand side of equation (5.1), after applying the soft-graviton theorem and the KLT-relation, is given in equation (5.2). At $\mathcal{O}(1 / \epsilon)$, the terms in equation (5.2) are given by $P_{1-6}$, where

$$
\begin{aligned}
& P_{1}=(-1)^{n} \sum_{t=1}^{n-2} \sum_{\sigma, \rho \in S_{n-3}} \frac{1}{\epsilon} \frac{[t, n+1]}{\langle t, n+1\rangle} \mathcal{A}_{n}\left[\frac{1}{2} \tilde{\nabla}_{n+1, t}^{2} \mathcal{S}[\sigma \mid \rho]_{p_{n-1}}\right] \tilde{\mathcal{A}}_{n}, \\
& P_{2}=(-1)^{n} \sum_{t=1}^{n-2} \sum_{\sigma, \rho \in S_{n-3}} \frac{1}{\epsilon} \frac{[t, n+1]}{\langle t, n+1\rangle}\left[\frac{1}{2} \tilde{\nabla}_{n+1, t}^{2} \mathcal{A}_{n}\right] \mathcal{S}[\sigma \mid \rho]_{p_{n-1}} \tilde{\mathcal{A}}_{n}, \\
& P_{3}=(-1)^{n} \sum_{t=1}^{n-2} \sum_{\sigma, \rho \in S_{n-3}} \frac{1}{\epsilon} \frac{[t, n+1]}{\langle t, n+1\rangle} \mathcal{A}_{n} \mathcal{S}[\sigma \mid \rho]_{p_{n-1}}\left[\frac{1}{2} \tilde{\nabla}_{n+1, t}^{2} \tilde{\mathcal{A}}_{n}\right], \\
& P_{4}=(-1)^{n} \sum_{t=1}^{n-2} \sum_{\sigma, \rho \in S_{n-3}} \frac{1}{\epsilon} \frac{[t, n+1]}{\langle t, n+1\rangle}\left[\tilde{\nabla}_{n+1, t} \mathcal{A}_{n}\right]\left[\tilde{\nabla}_{n+1, t} \mathcal{S}[\sigma \mid \rho]_{p_{n-1}}\right] \tilde{\mathcal{A}}_{n}, \\
& P_{5}=(-1)^{n} \sum_{t=1}^{n-2} \sum_{\sigma, \rho \in S_{n-3}} \frac{1}{\epsilon} \frac{[t, n+1]}{\langle t, n+1\rangle}\left[\tilde{\nabla}_{n+1, t} \mathcal{A}_{n}\right] \mathcal{S}[\sigma \mid \rho]_{p_{n-1}}\left[\tilde{\nabla}_{n+1, t} \tilde{\mathcal{A}}_{n}\right], \\
& P_{6}=(-1)^{n} \sum_{t=1}^{n-2} \sum_{\sigma, \rho \in S_{n-3}} \frac{1}{\epsilon} \frac{[t, n+1]}{\langle t, n+1\rangle} \mathcal{A}_{n}\left[\tilde{\nabla}_{n+1, t} \mathcal{S}[\sigma \mid \rho]_{p_{n-1}}\right]\left[\tilde{\nabla}_{n+1, t} \tilde{\mathcal{A}}_{n}\right] .
\end{aligned}
$$

Similarly, the right-hand side of equation (5.1) is given in equation (5.3), consisting of $Q_{1-6}$ and $R_{1,2}$. The non-universal terms $R_{1,2}$ are given in equations (5.4) and (5.5), with the 
residual terms being

$$
\begin{aligned}
& Q_{1}=(-1)^{n} \sum_{t=1}^{n-2} \sum_{\sigma, \rho \in S_{n-3}} \frac{1}{\epsilon} \frac{[t, n+1]\langle t, n-1\rangle\langle n, n+1\rangle}{\langle t, n+1\rangle\langle n+1, n-1\rangle\langle n, t\rangle} \mathcal{A}_{n}\left[\frac{1}{2} \tilde{\nabla}_{n+1, t}^{2} \mathcal{S}[\sigma \mid \rho]_{p_{n-1}}\right] \tilde{\mathcal{A}}_{n}, \\
& Q_{2}=(-1)^{n} \sum_{t=1}^{n-2} \sum_{\sigma, \rho \in S_{n-3}} \frac{1}{\epsilon} \frac{[t, n+1]\langle t, n-1\rangle\langle n, n+1\rangle}{\langle t, n+1\rangle\langle n+1, n-1\rangle\langle n, t\rangle}\left[\frac{1}{2} \tilde{\nabla}_{n+1, t}^{2} \mathcal{A}_{n}\right] \mathcal{S}[\sigma \mid \rho]_{p_{n-1}} \tilde{\mathcal{A}}_{n} \\
& Q_{3}=(-1)^{n} \sum_{t=1}^{n-2} \sum_{\sigma, \rho \in S_{n-3}} \frac{1}{\epsilon} \frac{[t, n+1]\langle n, t\rangle\langle n-1, n+1\rangle}{\langle t, n+1\rangle\langle n, n+1\rangle\langle n-1, t\rangle} \mathcal{A}_{n} \mathcal{S}[\sigma \mid \rho]_{p_{n-1}}\left[\frac{1}{2} \tilde{\nabla}_{n+1, t}^{2} \tilde{\mathcal{A}}_{n}\right] \\
& Q_{4}=(-1)^{n} \sum_{t=1}^{n-2} \sum_{\sigma, \rho \in S_{n-3}} \frac{1}{\epsilon} \frac{[t, n+1]\langle t, n-1\rangle\langle n, n+1\rangle}{\langle t, n+1\rangle\langle n+1, n-1\rangle\langle n, t\rangle}\left[\tilde{\nabla}_{n+1, t} \mathcal{A}_{n}\right]\left[\tilde{\nabla}_{n+1, t} \mathcal{S}[\sigma \mid \rho]_{p_{n-1}}\right] \tilde{\mathcal{A}}_{n}, \\
& Q_{5}=(-1)^{n} \sum_{t=1}^{n-2} \sum_{\sigma, \rho \in S_{n-3}} \frac{1}{\epsilon} \frac{[t, n+1]}{\langle t, n+1\rangle}\left[\tilde{\nabla}_{n+1, t}, \mathcal{A}_{n}\right] \mathcal{S}[\sigma \mid \rho]_{p_{n-1}}\left[\tilde{\nabla}_{n+1, t}, \tilde{\mathcal{A}}_{n}\right] \\
& Q_{6}=(-1)^{n} \sum_{t=1}^{n-2} \sum_{\sigma, \rho \in S_{n-3}} \frac{1}{\epsilon} \frac{[t, n+1]}{\langle t, n+1\rangle} \mathcal{A}_{n}\left[\tilde{\nabla}_{n+1, t}, \mathcal{S}[\sigma \mid \rho]_{p_{n-1}}\right]\left[\tilde{\nabla}_{n+1, t} \tilde{\mathcal{A}}_{n}\right]
\end{aligned}
$$

Note that

$$
\begin{aligned}
& P_{5}=Q_{5}, \\
& P_{6}=Q_{6},
\end{aligned}
$$

which reduces equation (5.1) to

$$
P_{1}+P_{2}+P_{3}+P_{4}=Q_{1}+Q_{2}+Q_{3}+Q_{4}+R_{1}+R_{2} .
$$

We can simplify this further by using that

$$
T_{1}=P_{1}+P_{2}+P_{4}-Q_{1}-Q_{2}-Q_{4} \quad \text { and } \quad T_{2}=P_{3}-Q_{3},
$$

which are the terms used in the main text, given in equations (5.6) and (5.7). With these manipulations, we find new, non-trivial relations for the non-universal part of the Yang-Mills amplitudes. The new relations, equations (5.9) and (5.10), have surprisingly simple forms.

\section{B Sub-subleading terms from effective operator}

The new contributions to equation (5.1) coming from the soft theorems for effective operators involve $\bar{S}_{\mathrm{YM}}^{(1)}$ and $\bar{S}_{\mathrm{YM}}^{(2)}$. We will denote the terms by $\bar{Q}_{2-6}$ as they resemble $Q_{2-6}$ in equations (A.8) to (A.12). In general, as $\bar{S}_{\mathrm{YM}}^{(1,2)}$ contain two different terms, we express $\bar{Q}_{2-6}$ as two terms, e.g. $\bar{Q}_{2}=\bar{Q}_{2}^{(t)}+\bar{Q}_{2}^{(n)}$. Note that $\bar{Q}_{5}$ is split into eight terms,

$$
\bar{Q}_{5}=\bar{Q}_{5}^{(n, n-1)}+\bar{Q}_{5}^{(n, t)}+\bar{Q}_{5}^{(t, n-1)}+\bar{Q}_{5}^{(t, t)}+\bar{Q}_{5}^{(L, n)}+\bar{Q}_{5}^{(L, t)}+\bar{Q}_{5}^{(R, n-1)}+\bar{Q}_{5}^{(R, t)} .
$$


The terms are

$$
\begin{aligned}
& \bar{Q}_{2}^{(t)}=\frac{(-1)^{n+1}}{\epsilon} \sum_{t=1}^{n-2} \sum_{\sigma, \rho \in S_{n-3}} \frac{[t, n+1]^{2}\langle t, n-1\rangle\langle n, n+1\rangle}{\langle n+1, n-1\rangle\langle n+1, t\rangle\langle n, t\rangle}\left[\tilde{\nabla}_{n+1, t} \overline{\mathcal{A}}_{n}^{(t)}\right] \mathcal{S}[\sigma \mid \rho]_{p_{n-1}} \tilde{\mathcal{A}}_{n}, \\
& \bar{Q}_{2}^{(n)}=\frac{(-1)^{n+1}}{\epsilon} \sum_{t=1}^{n-2} \sum_{\sigma, \rho \in S_{n-3}} \frac{[t, n+1]\langle t, n-1\rangle[n+1, n]}{\langle n+1, n-1\rangle\langle n, t\rangle}\left[\tilde{\nabla}_{n+1, t} \overline{\mathcal{A}}_{n}^{(n)}\right] \mathcal{S}[\sigma \mid \rho]_{p_{n-1}} \tilde{\mathcal{A}}_{n}, \\
& \bar{Q}_{3}^{(t)}=\frac{(-1)^{n+1}}{\epsilon} \sum_{t=1}^{n-2} \sum_{\sigma, \rho \in S_{n-3}} \frac{[t, n+1]^{2}\langle n, t\rangle\langle n-1, n+1\rangle}{\langle n+1, n\rangle\langle n+1, t\rangle\langle n-1, t\rangle} \mathcal{A}_{n} \mathcal{S}[\sigma \mid \rho]_{p_{n-1}}\left[\tilde{\nabla}_{n+1, t} \tilde{\mathcal{\mathcal { A }}}_{n}^{(t)}\right], \\
& \bar{Q}_{3}^{(n-1)}=\frac{(-1)^{n+1}}{\epsilon} \sum_{t=1}^{n-2} \sum_{\sigma, \rho \in S_{n-3}} \frac{[t, n+1][n+1, n-1]\langle n, t\rangle}{\langle n+1, n\rangle\langle n-1, t\rangle} \mathcal{A}_{n} \mathcal{S}[\sigma \mid \rho]_{p_{n-1}}\left[\tilde{\nabla}_{n+1, t} \tilde{\mathcal{\mathcal { A }}}_{n}^{(n-1)}\right] \text {. } \\
& \bar{Q}_{4}^{(n)}=\frac{(-1)^{n}}{\epsilon} \sum_{t=1}^{n-2} \sum_{\sigma, \rho \in S_{n-3}} \frac{[t, n+1]\langle t, n-1\rangle\langle n, n+1\rangle[n+1, n]}{\langle n+1, n-1\rangle\langle n, t\rangle\langle n+1, n\rangle} \overline{\mathcal{A}}_{n}^{(n)}\left[\tilde{\nabla}_{n+1, t} \mathcal{S}[\sigma \mid \rho]_{p_{n-1}}\right] \tilde{\mathcal{A}}_{n}, \\
& \bar{Q}_{4}^{(t)}=\frac{(-1)^{n}}{\epsilon} \sum_{t=1}^{n-2} \sum_{\sigma, \rho \in S_{n-3}} \frac{[t, n+1]\langle t, n-1\rangle\langle n, n+1\rangle[n+1, t]}{\langle n+1, n-1\rangle\langle n, t\rangle\langle n+1, t\rangle} \overline{\mathcal{A}}_{n}^{(t)}\left[\tilde{\nabla}_{n+1, t} \mathcal{S}[\sigma \mid \rho]_{p_{n-1}}\right] \tilde{\mathcal{A}}_{n}, \\
& \bar{Q}_{5}^{(n, n-1)}=\frac{(-1)^{n}}{\epsilon} \sum_{t=1}^{n-2} \sum_{\sigma, \rho \in S_{n-3}} \frac{[t, n+1]\langle n+1, t\rangle[n+1, n][n+1, n-1]}{\langle n+1, n\rangle\langle n+1, n-1\rangle} \overline{\mathcal{A}}_{n}^{(n)} \mathcal{S}[\sigma \mid \rho]_{p_{n-1}} \tilde{\mathcal{\mathcal { A }}}_{n}^{(n-1)}, \\
& \bar{Q}_{5}^{(n, t)}=\frac{(-1)^{n+1}}{\epsilon} \sum_{t=1}^{n-2} \sum_{\sigma, \rho \in S_{n-3}} \frac{[t, n+1]^{2}[n+1, n]}{\langle n+1, n\rangle} \overline{\mathcal{A}}_{n}^{(n)} \mathcal{S}[\sigma \mid \rho]_{p_{n-1}} \tilde{\overline{\mathcal{A}}}_{n}^{(t)}, \\
& \bar{Q}_{5}^{(t, n-1)}=\frac{(-1)^{n+1}}{\epsilon} \sum_{t=1}^{n-2} \sum_{\sigma, \rho \in S_{n-3}} \frac{[t, n+1]^{2}[n+1, n-1]}{\langle n+1, n-1\rangle} \overline{\mathcal{A}}_{n}^{(n)} \mathcal{S}[\sigma \mid \rho]_{p_{n-1}} \tilde{\overline{\mathcal{A}}}_{n}^{(n-1)}, \\
& \bar{Q}_{5}^{(t, t)}=\frac{(-1)^{n+1}}{\epsilon} \sum_{t=1}^{n-2} \sum_{\sigma, \rho \in S_{n-3}} \frac{[n+1, t]^{3}}{\langle n+1, t\rangle} \overline{\mathcal{A}}_{n}^{(t)} \mathcal{S}[\sigma \mid \rho]_{p_{n-1}} \tilde{\mathcal{\mathcal { A }}}_{n}^{(t)}, \\
& \bar{Q}_{5}^{(L, n)}=\frac{(-1)^{n}}{\epsilon} \sum_{t=1}^{n-2} \sum_{\sigma, \rho \in S_{n-3}} \frac{[t, n+1][n+1, n]}{\langle n+1, n\rangle} \overline{\mathcal{A}}_{n}^{(n)} \mathcal{S}[\sigma \mid \rho]_{p_{n-1}}\left[\tilde{\nabla}_{n+1, t} \tilde{\mathcal{A}}_{n}\right], \\
& \bar{Q}_{5}^{(L, t)}=\frac{(-1)^{n}}{\epsilon} \sum_{t=1}^{n-2} \sum_{\sigma, \rho \in S_{n-3}} \frac{[t, n+1][n+1, t]}{\langle n+1, t\rangle} \overline{\mathcal{A}}_{n}^{(t)} \mathcal{S}[\sigma \mid \rho]_{p_{n-1}}\left[\tilde{\nabla}_{n+1, t} \tilde{\mathcal{A}}_{n}\right], \\
& \bar{Q}_{5}^{(R, n-1)}=\frac{(-1)^{n+1}}{\epsilon} \sum_{t=1}^{n-2} \sum_{\sigma, \rho \in S_{n-3}} \frac{[t, n+1][n+1, n-1]}{\langle n+1, n-1\rangle}\left[\tilde{\nabla}_{n+1, t} \mathcal{A}_{n}\right] \mathcal{S}[\sigma \mid \rho]_{p_{n-1}} \tilde{\overline{\mathcal{A}}}_{n}^{(n-1)} \\
& \bar{Q}_{5}^{(R, t)}=\frac{(-1)^{n+1}}{\epsilon} \sum_{t=1}^{n-2} \sum_{\sigma, \rho \in S_{n-3}} \frac{[t, n+1][n+1, t]}{\langle n+1, t\rangle}\left[\tilde{\nabla}_{n+1, t} \mathcal{A}_{n}\right] \mathcal{S}[\sigma \mid \rho]_{p_{n-1}} \tilde{\mathcal{\mathcal { A }}}_{n}^{(t)} . \\
& \bar{Q}_{6}^{(n-1)}=\frac{(-1)^{n+1}}{\epsilon} \sum_{t=1}^{n-2} \sum_{\sigma, \rho \in S_{n-3}} \frac{[t, n+1][n+1, n-1]}{\langle n+1, n-1\rangle} \mathcal{A}_{n}\left[\tilde{\nabla}_{n+1, t} \mathcal{S}[\sigma \mid \rho]_{p_{n-1}}\right] \tilde{\overline{\mathcal{A}}}_{n}^{(n-1)} \\
& \bar{Q}_{6}^{(t)}=\frac{(-1)^{n+1}}{\epsilon} \sum_{t=1}^{n-2} \sum_{\sigma, \rho \in S_{n-3}} \frac{[t, n+1][n+1, t]}{\langle n+1, t\rangle} \mathcal{A}_{n}\left[\tilde{\nabla}_{n+1, t} \mathcal{S}[\sigma \mid \rho]_{p_{n-1}}\right] \tilde{\overline{\mathcal{A}}}_{n}^{(t)} .
\end{aligned}
$$


The strategy now is to group $\bar{Q}_{2}^{(t)}$ and $\bar{Q}_{4}^{(t)}$ with $\bar{Q}_{5}^{(L, t)}$. Notice that each term has a derivative and comes with the superscript $(t)$. We apply the Schouten identity on the spinor brackets in the first two terms to match the last one. This produces a 'total' derivative called $\bar{Q}_{L}^{(t)}$ and an extra term, which we call $\bar{Q}_{S}^{(t 1)}$. We do the same for others operators, finding the following rearragements

$$
\begin{aligned}
\bar{Q}_{2}^{(t)}+\bar{Q}_{4}^{(t)}+\bar{Q}_{5}^{(L, t)} & =\bar{Q}_{L}^{(t)}+\bar{Q}_{S}^{(t 1)}, \\
\bar{Q}_{2}^{(n)}+\bar{Q}_{4}^{(n)}+\bar{Q}_{5}^{(L, n)} & =\bar{Q}_{L}^{(n)}+\bar{Q}_{S}^{(n)}, \\
\bar{Q}_{3}^{(t)}+\bar{Q}_{6}^{(t)}+\bar{Q}_{5}^{(R, t)} & =\bar{Q}_{R}^{(t)}+\bar{Q}_{S}^{(t 2)}, \\
\bar{Q}_{3}^{(n-1)}+\bar{Q}_{6}^{(n-1)}+\bar{Q}_{5}^{(R, n-1)} & =\bar{Q}_{R}^{(n-1)}+\bar{Q}_{S}^{(n-1)},
\end{aligned}
$$

where

$$
\begin{aligned}
& \bar{Q}_{L}^{(k)}=\frac{(-1)^{n+1}}{\epsilon} \sum_{t=1}^{n-2} \sum_{\sigma, \rho \in S_{n-3}}(-1)[t, n+1] \frac{[n+1, k]}{\langle n+1, k\rangle} \tilde{\nabla}_{n+1, t}\left[\overline{\mathcal{A}}_{n}^{(k)} \mathcal{S}[\sigma \mid \rho]_{p_{n-1}} \tilde{\mathcal{A}}_{n}\right], \\
& \bar{Q}_{R}^{(k)}=\frac{(-1)^{n+1}}{\epsilon} \sum_{t=1}^{n-2} \sum_{\sigma, \rho \in S_{n-3}}(-1)[t, n+1] \frac{[n+1, k]}{\langle n+1, k\rangle} \tilde{\nabla}_{n+1, t}\left[\mathcal{A}_{n} \mathcal{S}[\sigma \mid \rho]_{p_{n-1}} \tilde{\mathcal{A}}_{n}^{(k)}\right] .
\end{aligned}
$$

The extra terms are

$$
\begin{aligned}
\bar{Q}_{S}^{(t 1)}= & \frac{(-1)^{n+1}}{\epsilon} \sum_{t=1}^{n-2} \sum_{\sigma, \rho \in S_{n-3}} \frac{[t, n+1]^{2}\langle n-1, n\rangle}{\langle n+1, n-1\rangle\langle n, t\rangle} \tilde{\nabla}_{n+1, t}\left[\overline{\mathcal{A}}_{n}^{(t)} \mathcal{S}[\sigma \mid \rho]_{p_{n-1}}\right] \tilde{\mathcal{A}}_{n}, \\
\bar{Q}_{S}^{(n)}= & \frac{(-1)^{n}}{\epsilon} \sum_{t=1}^{n-2} \sum_{\sigma, \rho \in S_{n-3}} \frac{[t, n+1][n, n-1]\langle t, n+1\rangle\langle n, n-1\rangle}{\langle n+1, n-1\rangle\langle n, t\rangle\langle n+1, n\rangle} \tilde{\nabla}_{n+1, t}\left[\overline{\mathcal{A}}_{n}^{(n)} \mathcal{S}[\sigma \mid \rho]_{p_{n-1}}\right] \tilde{\mathcal{A}}_{n}, \\
\bar{Q}_{S}^{(t 2)}= & \frac{(-1)^{n+1}}{\epsilon} \sum_{t=1}^{n-2} \sum_{\sigma, \rho \in S_{n-3}} \frac{[t, n+1]^{2}\langle n, n-1\rangle}{\langle n+1, n\rangle\langle n-1, t\rangle} \mathcal{A}_{n} \mathcal{S}[\sigma \mid \rho]_{p_{n-1}}\left[\tilde{\nabla}_{n+1, t} \tilde{\overline{\mathcal{A}}}_{n}^{(t)}\right] \\
\bar{Q}_{S}^{(n-1)}= & \frac{(-1)^{n}}{\epsilon-2} \sum_{t=1}^{n} \sum_{\sigma, \rho \in S_{n-3}} \\
& \times \frac{[t, n+1][n+1, n-1]\langle n, n-1\rangle\langle t, n+1\rangle}{\langle n+1, n\rangle\langle n-1, t\rangle\langle n+1, n-1\rangle} \mathcal{A}_{n} \mathcal{S}[\sigma \mid \rho]_{p_{n-1}}\left[\tilde{\nabla}_{n+1, t} \tilde{\mathcal{\mathcal { A }}}_{n}^{(n-1)}\right] .
\end{aligned}
$$

We group the terms together,

$$
\begin{aligned}
& \bar{Q}_{S}=\bar{Q}_{S}^{(n)}+\bar{Q}_{S}^{(t 1)}+\bar{Q}_{S}^{(t 2)}+\bar{Q}_{S}^{(n-1)}, \\
& \bar{Q}_{5^{\prime}}=\bar{Q}_{5}^{(n, n-1)}+\bar{Q}_{5}^{(n, t)}+\bar{Q}_{5}^{(t, n-1)}+\bar{Q}_{5}^{(t, t)}, \\
& \bar{Q}_{B}=\bar{Q}_{L}^{(t)}+\bar{Q}_{L}^{(n)}+\bar{Q}_{R}^{(t)}+\bar{Q}_{R}^{(n-1)} .
\end{aligned}
$$

We also have contributions from the higher-order terms in the expansion of the kernel. We group them as

$$
\bar{Q}_{K}=T_{1}^{\prime}+T_{2}^{\prime}+T_{3}^{\prime}+T_{4}^{\prime}+\bar{U}_{1}^{\prime}+\bar{U}_{2}^{\prime}
$$


The two last terms are related to $U_{1}$ and $U_{2}$ in equations (6.10) and (6.11) as

$$
\begin{aligned}
& \bar{U}_{1}^{\prime}=\epsilon c_{1} s_{t, n+1} U_{1}, \\
& \bar{U}_{2}^{\prime}=\epsilon c_{1} s_{t, n+1} U_{2},
\end{aligned}
$$

where the factor $s_{t, n+1}$ is understood to be inside the sum over $t$.

The other terms are

$$
\begin{aligned}
& T_{1}^{\prime}=\frac{(-1)^{n+1}}{\epsilon} c_{1} \sum_{t=1}^{n-2} \sum_{\sigma, \rho \in S_{n-3}} \frac{[t, n+1]^{2}\langle n, t\rangle}{\langle n, n+1\rangle} \mathcal{A}_{n} \mathcal{S}[\sigma \mid \rho]_{p_{n-1}}\left[\tilde{\nabla}_{n+1, t} \tilde{\mathcal{A}}_{n}\right], \\
& T_{2}^{\prime}=\frac{(-1)^{n+1}}{\epsilon} c_{1} \sum_{t=1}^{n-2} \sum_{\sigma, \rho \in S_{n-3}} \frac{[t, n+1]^{2}\langle n-1, t\rangle}{\langle n-1, n+1\rangle}\left[\tilde{\nabla}_{n+1, t} \mathcal{A}_{n}\right] \mathcal{S}[\sigma \mid \rho]_{p_{n-1}} \tilde{\mathcal{A}}_{n}, \\
& T_{3}^{\prime}=\frac{(-1)^{n+1}}{\epsilon} c_{1} \sum_{t=1}^{n-2} \sum_{\sigma, \rho \in S_{n-3}} \frac{[t, n+1]^{2}\langle n-1, t\rangle}{\langle n-1, n+1\rangle} \mathcal{A}_{n}\left[\tilde{\nabla}_{n+1, t} \mathcal{S}[\sigma \mid \rho]_{p_{n-1}}\right] \tilde{\mathcal{A}}_{n}, \\
& T_{4}^{\prime}=\frac{(-1)^{n}}{\epsilon} c_{2} \sum_{t=1}^{n-2} \sum_{\sigma, \rho \in S_{n-3}} \frac{[t, n+1]^{3}\langle n, t\rangle\langle t, n-1\rangle\langle t, n+1\rangle}{\langle n, n+1\rangle\langle n+1, n-1\rangle}\left[\mathcal{A}_{n} \mathcal{S}[\sigma \mid \rho]_{p_{n-1}} \tilde{\mathcal{A}}_{n}\right] .
\end{aligned}
$$

We can rewrite $T_{1-3}^{\prime}$ using the Schouten identity to

$$
T_{1}^{\prime}+T_{2}^{\prime}+T_{3}^{\prime}=T^{\prime}+T_{s}^{\prime},
$$

where

$$
\begin{aligned}
& T^{\prime}=\frac{(-1)^{n+1}}{\epsilon} c_{1} \sum_{t=1}^{n-2} \sum_{\sigma, \rho \in S_{n-3}} \frac{[t, n+1]^{2}\langle n-1, t\rangle}{\langle n-1, n+1\rangle} \tilde{\nabla}_{n+1, t}\left[\mathcal{A}_{n} \mathcal{S}[\sigma \mid \rho]_{p_{n-1}} \tilde{\mathcal{A}}_{n}\right], \\
& T_{s}^{\prime}=\frac{(-1)^{n}}{\epsilon} c_{1} \sum_{t=1}^{n-2} \sum_{\sigma, \rho \in S_{n-3}} \frac{[t, n+1]^{2}\langle n, n-1\rangle\langle t, n+1\rangle}{\langle n, n+1\rangle\langle n+1, n-1\rangle} \mathcal{A}_{n} \mathcal{S}[\sigma \mid \rho]_{p_{n-1}}\left[\tilde{\nabla}_{n+1, t} \tilde{\mathcal{A}}_{n}\right] .
\end{aligned}
$$

In total, we have that

$$
\bar{Q}=\bar{Q}_{5^{\prime}}+\bar{Q}_{B}+\bar{Q}_{S}+\bar{Q}_{K} .
$$

Open Access. This article is distributed under the terms of the Creative Commons Attribution License (CC-BY 4.0), which permits any use, distribution and reproduction in any medium, provided the original author(s) and source are credited.

\section{References}

[1] F. Bloch and A. Nordsieck, Note on the Radiation Field of the electron, Phys. Rev. 52 (1937) 54 [INSPIRE].

[2] A. Nordsieck, The Low Frequency Radiation of a Scattered Electron, Phys. Rev. 52 (1937) 59 [INSPIRE]. 
[3] F.E. Low, Scattering of light of very low frequency by systems of spin 1/2, Phys. Rev. 96 (1954) 1428 [inSPIRE].

[4] M. Gell-Mann and M.L. Goldberger, Scattering of low-energy photons by particles of spin 1/2, Phys. Rev. 96 (1954) 1433 [inSPIRE].

[5] F.E. Low, Bremsstrahlung of very low-energy quanta in elementary particle collisions, Phys. Rev. 110 (1958) 974 [INSPIRE].

[6] D.R. Yennie, S.C. Frautschi and H. Suura, The infrared divergence phenomena and high-energy processes, Annals Phys. 13 (1961) 379 [INSPIRE].

[7] T. Kinoshita, Mass singularities of Feynman amplitudes, J. Math. Phys. 3 (1962) 650 [INSPIRE].

[8] T.D. Lee and M. Nauenberg, Degenerate Systems and Mass Singularities, Phys. Rev. 133 (1964) B1549 [INSPIRE].

[9] S. Weinberg, Photons and Gravitons in S-Matrix Theory: Derivation of Charge Conservation and Equality of Gravitational and Inertial Mass, Phys. Rev. 135 (1964) B1049 [InSPIRE].

[10] S. Weinberg, Infrared photons and gravitons, Phys. Rev. 140 (1965) B516 [inSPIRE].

[11] T.H. Burnett and N.M. Kroll, Extension of the low soft photon theorem, Phys. Rev. Lett. 20 (1968) 86 [INSPIRE].

[12] J.S. Bell and R. Van Royen, On the low-burnett-kroll theorem for soft-photon emission, Nuovo Cim. A 60 (1969) 62 [inSPIRE].

[13] E. Laenen, G. Stavenga and C.D. White, Path integral approach to eikonal and next-to-eikonal exponentiation, JHEP 03 (2009) 054 [arXiv:0811.2067] [INSPIRE].

[14] E. Laenen, L. Magnea, G. Stavenga and C.D. White, Next-to-Eikonal Corrections to Soft Gluon Radiation: A Diagrammatic Approach, JHEP 01 (2011) 141 [arXiv:1010.1860] [INSPIRE].

[15] C.D. White, Factorization Properties of Soft Graviton Amplitudes, JHEP 05 (2011) 060 [arXiv: 1103.2981] [INSPIRE].

[16] F. Cachazo and A. Strominger, Evidence for a New Soft Graviton Theorem, arXiv: 1404.4091 [INSPIRE].

[17] H. Bondi, M.G.J. van der Burg and A.W.K. Metzner, Gravitational waves in general relativity. 7. Waves from axisymmetric isolated systems, Proc. Roy. Soc. Lond. A 269 (1962) 21 [INSPIRE].

[18] R.K. Sachs, Gravitational waves in general relativity. 8. Waves in asymptotically flat space-times, Proc. Roy. Soc. Lond. A 270 (1962) 103 [InSPIRE].

[19] A. Strominger, On BMS Invariance of Gravitational Scattering, JHEP 07 (2014) 152 [arXiv: 1312.2229] [INSPIRE].

[20] T. He, V. Lysov, P. Mitra and A. Strominger, BMS supertranslations and Weinberg's soft graviton theorem, JHEP 05 (2015) 151 [arXiv: 1401.7026] [INSPIRE].

[21] D. Kapec, V. Lysov, S. Pasterski and A. Strominger, Semiclassical Virasoro symmetry of the quantum gravity $\mathcal{S}$-matrix, JHEP 08 (2014) 058 [arXiv: 1406.3312] [INSPIRE].

[22] A. Strominger, Lectures on the Infrared Structure of Gravity and Gauge Theory, arXiv: 1703.05448 [INSPIRE]. 
[23] M. Campiglia and A. Laddha, Sub-subleading soft gravitons and large diffeomorphisms, JHEP 01 (2017) 036 [arXiv: 1608.00685] [INSPIRE].

[24] M. Campiglia and A. Laddha, Sub-subleading soft gravitons: New symmetries of quantum gravity?, Phys. Lett. B 764 (2017) 218 [arXiv:1605.09094] [INSPIRE].

[25] Z. Bern, S. Davies and J. Nohle, On Loop Corrections to Subleading Soft Behavior of Gluons and Gravitons, Phys. Rev. D 90 (2014) 085015 [arXiv:1405.1015] [INSPIRE].

[26] S. He, Y.-t. Huang and C. Wen, Loop Corrections to Soft Theorems in Gauge Theories and Gravity, JHEP 12 (2014) 115 [arXiv:1405.1410] [INSPIRE].

[27] S. Atul Bhatkar and B. Sahoo, Subleading Soft Theorem for arbitrary number of external soft photons and gravitons, JHEP 01 (2019) 153 [arXiv: 1809.01675] [INSPIRE].

[28] B.U.W. Schwab and A. Volovich, Subleading Soft Theorem in Arbitrary Dimensions from Scattering Equations, Phys. Rev. Lett. 113 (2014) 101601 [arXiv:1404.7749] [INSPIRE].

[29] N. Afkhami-Jeddi, Soft Graviton Theorem in Arbitrary Dimensions, arXiv:1405.3533 [INSPIRE].

[30] M. Zlotnikov, Sub-sub-leading soft-graviton theorem in arbitrary dimension, JHEP 10 (2014) 148 [arXiv: 1407.5936] [INSPIRE].

[31] C. Kalousios and F. Rojas, Next to subleading soft-graviton theorem in arbitrary dimensions, JHEP 01 (2015) 107 [arXiv: 1407.5982] [INSPIRE].

[32] F. Cachazo, S. He and E.Y. Yuan, Scattering equations and Kawai-Lewellen-Tye orthogonality, Phys. Rev. D 90 (2014) 065001 [arXiv:1306.6575] [INSPIRE].

[33] F. Cachazo, S. He and E.Y. Yuan, Scattering of Massless Particles in Arbitrary Dimensions, Phys. Rev. Lett. 113 (2014) 171601 [arXiv:1307.2199] [InSPIRE].

[34] F. Cachazo, S. He and E.Y. Yuan, Scattering of Massless Particles: Scalars, Gluons and Gravitons, JHEP 07 (2014) 033 [arXiv:1309.0885] [INSPIRE].

[35] Z. Bern, J.J.M. Carrasco and H. Johansson, Perturbative Quantum Gravity as a Double Copy of Gauge Theory, Phys. Rev. Lett. 105 (2010) 061602 [arXiv: 1004.0476] [INSPIRE].

[36] Z. Bern, J.J. Carrasco, M. Chiodaroli, H. Johansson and R. Roiban, The Duality Between Color and Kinematics and its Applications, arXiv:1909.01358 [INSPIRE].

[37] H. Kawai, D.C. Lewellen and S.H.H. Tye, A Relation Between Tree Amplitudes of Closed and Open Strings, Nucl. Phys. B 269 (1986) 1 [inSPIRE].

[38] R. Britto, F. Cachazo and B. Feng, New recursion relations for tree amplitudes of gluons, Nucl. Phys. B 715 (2005) 499 [hep-th/0412308] [INSPIRE].

[39] R. Britto, F. Cachazo, B. Feng and E. Witten, Direct proof of tree-level recursion relation in Yang-Mills theory, Phys. Rev. Lett. 94 (2005) 181602 [hep-th/0501052] [INSPIRE].

[40] Y.-J. Du, B. Feng, C.-H. Fu and Y. Wang, Note on Soft Graviton theorem by KLT Relation, JHEP 11 (2014) 090 [arXiv: 1408.4179] [INSPIRE].

[41] H. Elvang, C.R.T. Jones and S.G. Naculich, Soft Photon and Graviton Theorems in Effective Field Theory, Phys. Rev. Lett. 118 (2017) 231601 [arXiv:1611.07534] [InSPIRE].

[42] Z. Bern, L.J. Dixon, M. Perelstein and J.S. Rozowsky, Multileg one loop gravity amplitudes from gauge theory, Nucl. Phys. B 546 (1999) 423 [hep-th/9811140] [INSPIRE]. 
[43] N.E.J. Bjerrum-Bohr, P.H. Damgaard, B. Feng and T. Sondergaard, Proof of Gravity and Yang-Mills Amplitude Relations, JHEP 09 (2010) 067 [arXiv:1007.3111] [INSPIRE].

[44] M. Bianchi, S. He, Y.-t. Huang and C. Wen, More on Soft Theorems: Trees, Loops and Strings, Phys. Rev. D 92 (2015) 065022 [arXiv:1406.5155] [InSPIRE].

[45] N.E.J. Bjerrum-Bohr, String theory and the mapping of gravity into gauge theory, Phys. Lett. B 560 (2003) 98 [hep-th/0302131] [INSPIRE].

[46] N.E.J. Bjerrum-Bohr, Generalized string theory mapping relations between gravity and gauge theory, Nucl. Phys. B 673 (2003) 41 [hep-th/0305062] [INSPIRE].

[47] N.E.J. Bjerrum-Bohr and K. Risager, String theory and the KLT-relations between gravity and gauge theory including external matter, Phys. Rev. D 70 (2004) 086011 [hep-th/0407085] [INSPIRE].

[48] Y. Hamada and G. Shiu, Infinite Set of Soft Theorems in Gauge-Gravity Theories as Ward-Takahashi Identities, Phys. Rev. Lett. 120 (2018) 201601 [arXiv:1801.05528] [INSPIRE].

[49] Z.-Z. Li, H.-H. Lin and S.-Q. Zhang, Infinite Soft Theorems from Gauge Symmetry, Phys. Rev. D 98 (2018) 045004 [arXiv: 1802.03148] [INSPIRE].

[50] N.E.J. Bjerrum-Bohr, P.H. Damgaard, B. Feng and T. Sondergaard, New Identities among Gauge Theory Amplitudes, Phys. Lett. B 691 (2010) 268 [arXiv:1006.3214] [INSPIRE].

[51] L.A. Barreiro and R. Medina, The origin of the KLT relations and nonlinear relations for Yang-Mills amplitudes, Phys. Lett. B 803 (2020) 135299 [arXiv:1910.13519] [INSPIRE]. 